$$
\begin{aligned}
& \mathcal{W} \text { U } \\
& \text { Faculty of Economic Sciences } \\
& \text { Working Papers } \\
& \text { No. 25/2014 (142) } \\
& \text { JULIUSZ JABŁECKI } \\
& \text { RYSZARD KOKOSZCZYŃSKI } \\
& \text { PAWEŁ SAKOWSKI } \\
& \text { ROBERT ŚLEPACZUK } \\
& \text { PIOTR WÓJCIK }
\end{aligned}
$$

\title{
SIMPLE HEURISTICS FOR PRICING VIX OPTIONS
}




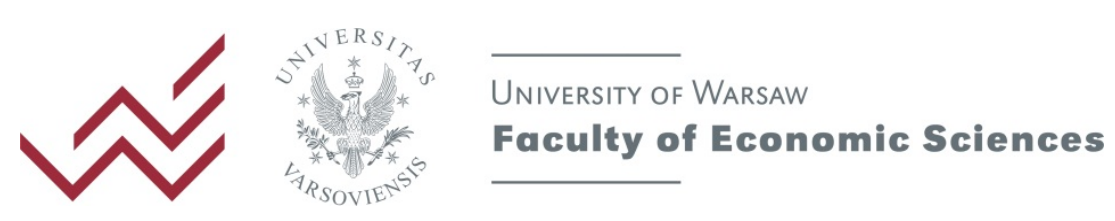

\title{
Simple heuristics for pricing VIX options
}

\author{
JULIUSZ JABLECKI \\ Faculty of Economic Sciences \\ University of Warsaw \\ National Bank of Poland \\ e-mail: jjablecki@wne.uw.edu.pl \\ PAWel SAKOWSKI \\ Faculty of Economic Sciences \\ University of Warsaw
}

\author{
RYSZARD KOKOSZCZYŃSKI \\ Faculty of Economic Sciences \\ University of Warsaw \\ National Bank of Poland
}

ROBERT ŚLEPACZUK

Faculty of Economic Sciences

University of Warsaw

\author{
Piotr WóJCIK \\ Faculty of Economic Sciences \\ University of Warsaw
}

\begin{abstract}
The article presents a simple parameterization of the volatility surface for options on the S\&P 500 volatility index, VIX. Specifically, we document the following features of VIX implied volatility: (i) VIX at-the-money (ATM) implied volatility correlates strongly with the volatility skew in S\&P 500 options; (ii) VIX ATM implied volatility declines exponentially with options' time to expiry; (iii) a SABR-type model can be used to model the smile observed in VIX options. These observations lead to simple heuristics for quoting prices (in terms of implied volatility) of VIX options with almost arbitrary strike and expiry, obtaining values that are reasonably close to market levels.
\end{abstract}

\section{Keywords:}

VIX, VIX options, implied volatility surface

\author{
JEL: \\ G12, G13
}

\section{Acknowledgments:}

The present paper is part of a research project "Term structure of VIX futures and the impact of volatility of volatility on other asset classes" supported by National Science Center grant number 2011/03/B/HS4/02298. We thank Arun Verma from Bloomberg for insightful comments and Maneesh Deshpande from Barclays for sharing parts of his own work on the topic. We also thank the participants of the Warsaw International Economic Meeting 2014 for helpful discussions. All views and remaining mistakes are our own. 


\section{Introduction}

VIX options are European options cash-settled to the value of the VIX index which synthetically captures the volatility implied from 30-day options on S\&P 500. As "derivatives of derivatives" VIX options are one of the most recent and sophisticated additions to the family tree of volatility products. Although VIX options were originally proposed alongside the introduction of the VIX index itself, i.e. as early as 1993 (Whaley, 1993), they were not launched until February 2006, and their popularity has only started growing with the advent of the recent global financial crisis. ${ }^{1}$ By now the market for VIX options has grown to an open interest of over 9 million contracts daily with total vega of about USD 200 million and strikes ranging from $70 \%$ to $300 \%$ of the index level (depending on expiry). From an economic point of view, VIX options allow investors to hedge their implicit or explicit volatility positions (derived e.g. from exposure to S\&P 500 or any closely correlated assets) as well as express directional views on volatility moves in a more risk-controlled way than allowed by e.g. VIX futures (see e.g. Moran and Dash, 2007 on the benefits of investing in VIX options). However, despite these virtues and apparent growth in the market for VIX options, the dedicated literature still remains relatively scarce.

Whaley (1993), the originator of the VIX index, was the first to consider pricing VIX options and suggested using the traditional Black (1976) model, with VIX futures as the underlying. Unfortunately, the shortcoming of Black's model is that it treats volatility as a constant, and in any case non-stochastic parameter, which is difficult to square with empirical observations. Thus, e.g. Grünbichler and Longstaff (1996) have adapted a simple stochastic process (similar to the one considered by Hull and White, 1987) to describe the evolution of the volatility index, obtaining closed-form formulas for prices of VIX options. As argued by Sepp (2008), the weakness of both approaches is that they separate the problem of pricing VIX options from the actual evolution of S\&P 500 volatility, and as a result they can miss-specify the vega (volatility of volatility) risk of VIX options which is crucial in hedging option portfolios and pricing more exotic products. A breakthrough in thinking about pricing volatility derivatives comes with the work of Derman, Demeterfi, Kamal, and Zou (1999) and Carr and Madan (2001) who showed that a position in volatility (variance) can be replicated in a model-free and essentially static way by taking a position in the whole available option chain. These ideas laid the groundwork for the refinement of the methodology behind the calculation of the VIX index (CBOE, 2003) thus allowing the development of a more robust

\footnotetext{
${ }^{1}$ The lag was largely due to the fact that a precondition for the development of a liquid options market is the existence of a liquid market in the underlying instrument - in this case VIX futures - needed to delta hedge the options. VIX futures did not start trading until 2004, when the methodology for the calculation of the VIX index was significantly improved following the development of the theory behind the replication of variance swaps. See esp. Dupire (2004), Derman, Demeterfi, Kamal, and Zou (1999) and Carr and Madan (2001) on replicating variance swap payoff, as well as CBOE (2003) on the new VIX methodology.
} 
theory of pricing volatility options that ensured consistency between option prices and the costs of their replication (Gatheral, 2008; Sepp, 2008; Lin and Chang, 2010 or Cont and Kokholm, 2011).

The goal of this article is much more modest. We propose to add to the current - mathematically sophisticated literature on VIX options - a set of simple heuristics, or empiricallygrounded intuitions, capturing the key features of VIX volatility surface, and providing market players with some pricing benchmarks. Specifically, we show that: (i) VIX at-the-money (ATM) implied volatility correlates strongly with the volatility skew in S\&P 500 options and we provide some theoretical justification for this relationship; (ii) VIX ATM implied volatility declines exponentially with options' time to expiry; (iii) a SABR-type model a la Hagan, Kumar, Lesniewski, and Woodward (2002) can be used to model the smile observed in VIX options. These observations lead to simple heuristics for quoting prices (in terms of implied volatility) of VIX options with almost arbitrary strike and expiry, obtaining values that generally lie with market bid-ask spreads. Clearly, the proposed parameterization is based on simple empirical observations rather than no-arbitrage conditions, and hence cannot be a substitute for more rigorous modeling approaches along the lines suggested by Gatheral (2008), Cont and Kokholm (2011) or Lin and Chang (2010). Nevertheless, we believe it can be a useful proxy, helpful especially for market practitioners interested in determining the viability of investing in VIX options in the long term, for which no actual VIX options prices are unavailable and back-casting them using sophisticated fully-fledged models could be resource and time intensive. ${ }^{2}$

\section{S\&P 500, VIX and options on both indices}

S\&P 500 (abbreviated henceforth by its ticker "SPX") is a stock market index of 500 largest companies listed on NYSE or NASDAQ exchanges. VIX is a measure of expected stock market volatility calculated on the basis of SPX options prices. Originally VIX was simply calculated as an arithmetic average of volatilities implied from the eight most liquid contracts - four calls and four puts. Since 2003 the calculation is based on a model-free statically replicating the payoff of a variance swap contract and utilizing all available contracts with a given expiry (Carr and Wu, 2006; CBOE, 2003). The key virtue of the new method is that it allows market participants to replicate or hedge their exposure to implied volatility. VIX represents simply market's expectation of the annualized standard deviation of S\&P 500 over the next month. As such, VIX is not directly investable i.e. there is no portfolio of assets

\footnotetext{
${ }^{2}$ This idea was suggested to us by Maneesh Deshpande whose team at Barclays developed a similar model to the one presented below (Deshpande, Bhatia, and Goyal, 2011). Our approach differs from theirs in that it goes to a full stochastic volatility model and models the VIX skew using SABR.
} 
Figure 1: Open interest in VIX derivatives

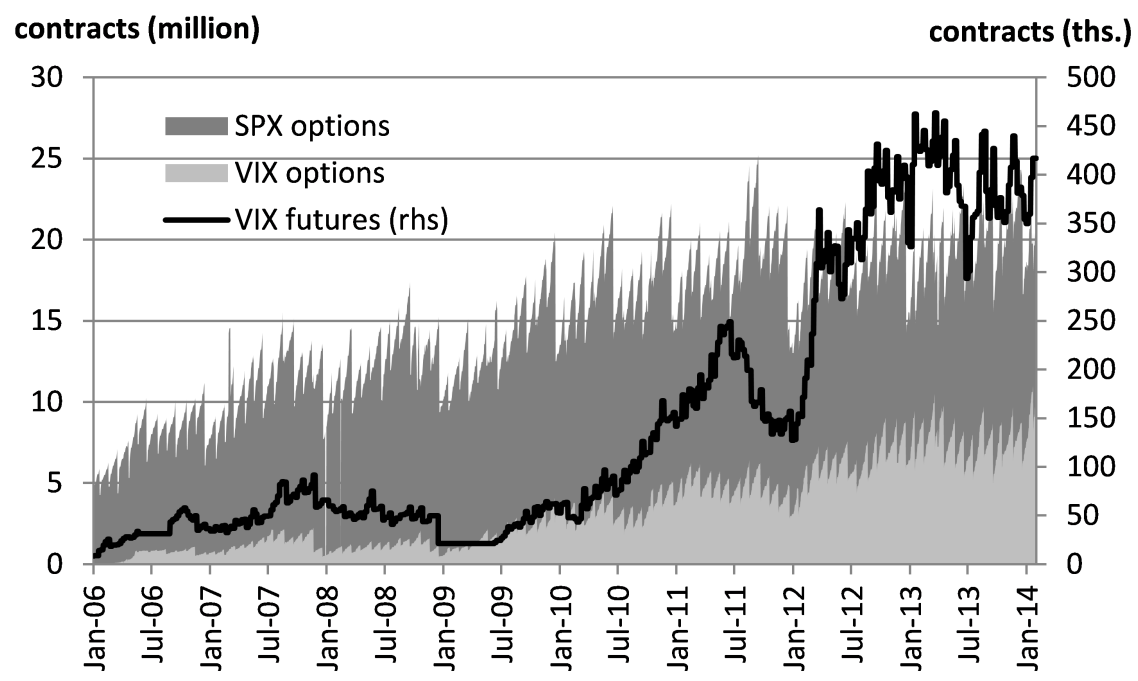

Source: Bloomberg, CBOE.

worth the VIX every day. ${ }^{3}$ However, VIX futures - i.e. futures contracts which settle to the value of the VIX on a given future expiration - introduced in 2004 by the CBOE are free from that shortcoming. VIX futures prices reflect each day market's expectation of SPX volatility that will be implied out of 30-day SPX options on a given settlement date in the future.

Given that VIX is not tradable, it is VIX futures that constitute the underlying instrument for VIX options. The latter were introduced in February 2006 as European contracts settled to the value of the VIX index, which the VIX future converges to. Although there are currently only six listed expiries for VIX options, corresponding to six following months (versus eight expiries for VIX futures), VIX options are the second most liquid group of option contracts listed on CBOE/CFE, slowly approaching SPX options in terms of open interest (Figure 1).

The natural starting point in investigating the pricing of VIX options is the celebrated Black-Scholes model (Black and Scholes 1973, Merton 1973 and Black 1976) which assumes that the underlying instrument $S$ obeys the following differential equation:

$$
\frac{d S_{t}}{S_{t}}=\mu d t+\sigma d Z_{t}
$$

where $\sigma$ denotes annualized standard deviation of $S$, and $Z$ is a Wiener process. Since it is not possible to invest directly in either SPX or VIX, the true underlying for option contracts

\footnotetext{
${ }^{3}$ The net profit on a contract that pays realized variance on a given underlying over a month (i.e. variance swap) will - after a week - be equal to the net profit accrued over that week and volatility expected over the remaining 3 weeks. In contrast, VIX each day expresses expected volatility over a rolling window of 30 days.
} 
Figure 2: S\&P 500 implied volatility surface as of January 30, 2014.

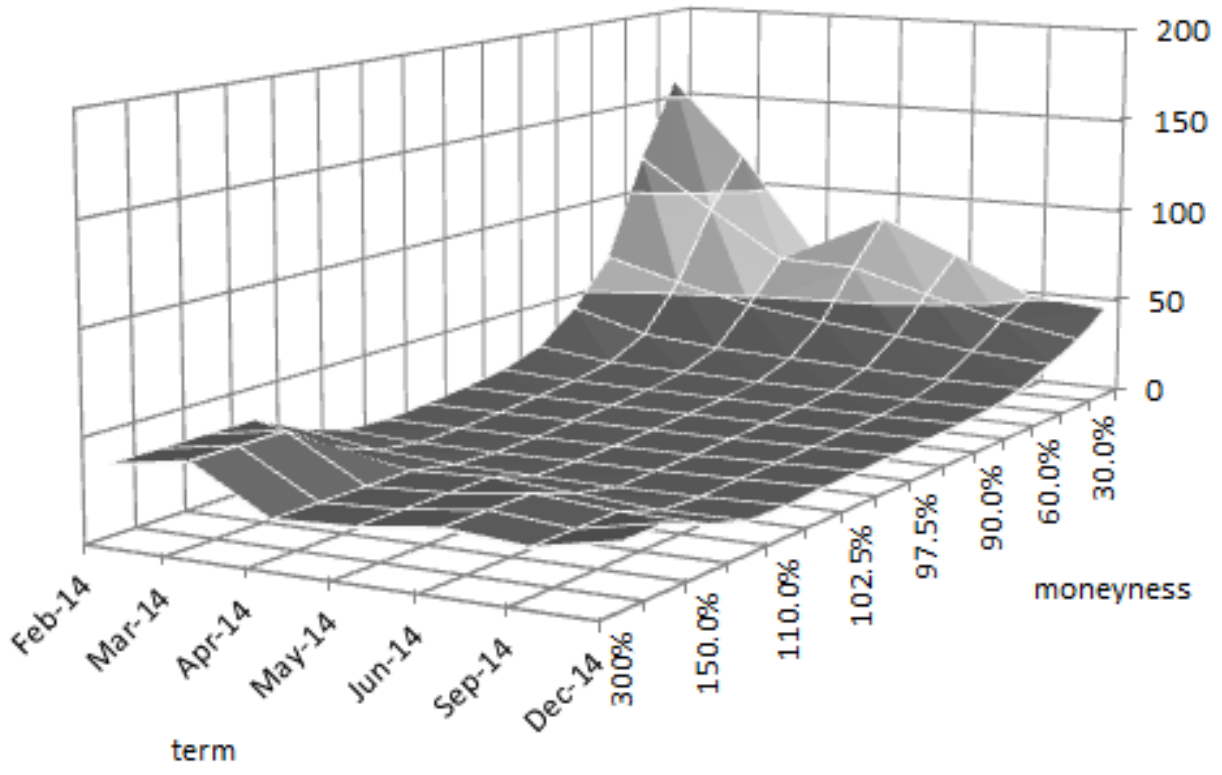

Source: Bloomberg, CBOE. For strike levels below ATM put-implied volatility is used and vice versa.

is a future contract $f_{t}(S)$, whose price converges to $S_{t}$. Hence, the price of a European call on $S$ with strike $K$ and expiry $T, C(K, T)$, is given by the expectation $\mathbb{E}\left(\max \left(S_{T}-K, 0\right)\right)$, or:

$$
C_{t}(K, T)=\exp (-r(T-t))\left[f_{t}(S) N\left(d_{+}\right)-K N\left(d_{-}\right)\right]
$$

where $r$ is the risk-free rate, $N(\cdot)$ is the standard normal CDF and

$$
d_{ \pm}=\frac{\log \left(f_{t} / K\right) \pm \frac{1}{2} \sigma^{2}(T-t)}{\sigma \sqrt{T-t}}
$$

The key assumption behind the Black-Scholes model is that $\sigma$ is an inherent, and moreover constant, feature of the underlying instrument $S$, not related to the specification of the option contract. In other words, irrespective of the expiry date and strike price specified in the option, the corresponding volatility levels should be the same.

As shown in Figures 2 and 3 for SPX and VIX this assumption is manifestly false. Implied volatilities of SPX and VIX options - i.e. parameters $\sigma$ that set the right-hand side of equation (2) equal to option prices quoted on the market - vary markedly with strike and time to expiration forming a non-trivial surface. This pattern clearly indicates that the Black-Scholes model - which foresees a flat volatility surface - cannot be used for consistent pricing of SPX or VIX options. However, due to the one-to-one correspondence between $C_{t}$ and $\sigma$, and the observability of the remaining parameters in equation (2), the Black-Scholes model is widely used as a convention for quoting options prices. Thus, in what follows 
Figure 3: VIX implied volatility surface as of January 30, 2014.

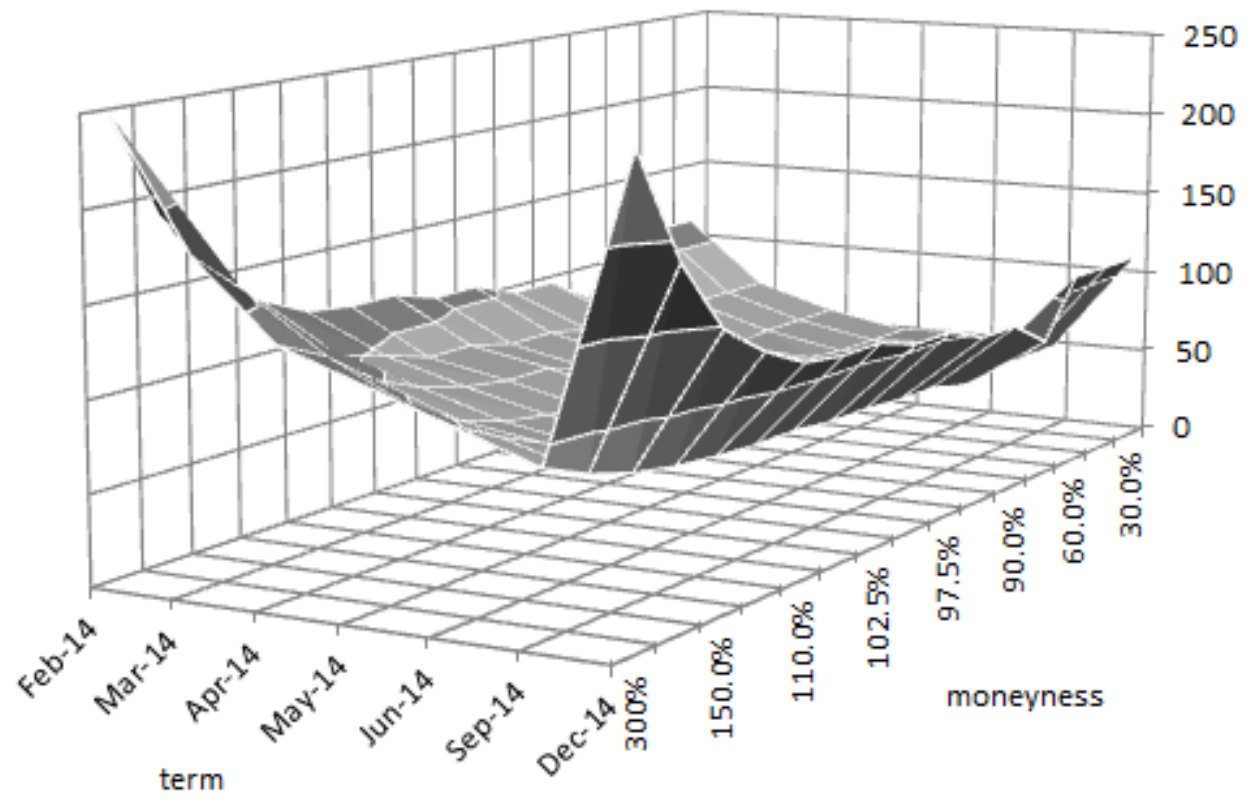

Source: Bloomberg, CBOE. For strike levels below ATM put-implied volatility is used and vice versa.

we present a number of simple empirical heuristics determining the behavior of the VIX volatility surface. While no substitute for rigorous formal models, these rules of thumb can help investors estimate reasonable prices of VIX options and assess the relative merits of volatility-based strategies.

\section{A simple model for pricing VIX options}

The existence of smiles - i.e. the empirically observed dependence of implied volatilities on strike and option expiry - can be elegantly accomodated in stochastic volatility models (Heston 1993; Hull and White 1987; see also an extensive overview e.g. in Alexander 2009, pp. 268-289). The advantage of these types of models is the possibility to capture both mean reversion of stock returns and volatility clustering. Hence, in what follows we present a fairly general formulation of a stochastic volatility model and discuss the relationship it implies between VIX and SPX implied volatilities. Then, once again starting from a general theoretical model, we show that VIX ATM implied volatility declines exponentially with the time to expiry of the contract. Finally, we calibrate a SABR-type model to VIX options prices quoted on the market, obtaining a full parameterization of the VIX volatility surface. Combined, these results can be used to provide reasonable "guesstimates" of VIX option prices based on the more liquid prices of SPX options. 


\subsection{Correlation between VIX and SPX skew}

Our first task is to identify the connection between SPX and VIX implied volatility. To do this we will need all the available information on SPX implied volatilities, captured in the smile. We start, however, from some theoretical background. Formally, volatility smile is defined as the partial derivative of an option's implied volatility with respect to absolute or relative strike ${ }^{4}$. Determining the smile thus requires the knowledge of the functional form of the implied volatility function (for a given expiry). To be able to move further we postulate a very general stochastic volatility model for the evolution of the underlying instrument $S_{t}$ and its volatility $v_{t}$, considered e.g. by Wilmott (2006) or Alexander $(2009)^{5}$ :

$$
\begin{aligned}
d S_{t} & =\mu_{t} S_{t} d t+\sqrt{v_{t}} S_{t} d Z_{1} \\
d v_{t} & =\alpha\left(v_{t}\right) d t+\eta \sqrt{v_{t}} \beta\left(v_{t}\right) d Z_{2} \\
d Z_{1} d Z_{2} & =\rho d t
\end{aligned}
$$

where $\mu_{t}$ is the deterministic drift, $\eta$ is the "volatility of volatility," while $\alpha(\cdot)$ and $\beta(\cdot)$ are any functions. The last condition says that the stochastic processes of the underlying and its variance have a fixed correlation coefficient $\rho$. In particular, setting $\eta=0$ reduces (4) to the Black-Scholes model. To simplify notation it is convenient to express our stochastic volatility model in terms of $\log$-moneyness, $x_{t}=\log \left(S_{t} / K\right)$, by applying the following change of variables:

$$
x_{t}:=\log \left(\frac{S_{t}}{F_{t}}\right)=\log \left(\frac{S_{t}}{S_{0} e^{r t}}\right)=\log \left(\frac{S_{t}}{S_{0}}\right)-r t
$$

Then, by Ito's lemma, $x_{t}$ evolved in risk-neutral measure according to

$$
d x_{t}=-\frac{1}{2} v_{t}+\sqrt{v_{t}} d Z_{1}
$$

Gatheral (2006) suggests the following orthogonalization of Wiener processes:

$$
d Z_{2}=\rho d Z_{1}+\sqrt{1-\rho^{2}} d \bar{Z}_{1}
$$

\footnotetext{
${ }^{4}$ See e.g. Gatheral (2006). The term "volatility smile" is often used in the literature in its broadest sense to describe the dependence of an option's implied volatility on strike (with a given time to expiration). However, since in some markets the smile does not necessarily look like a smile, the terms volatility skew or smirk are often used instead. Specifically, for SPX - in which case OTM puts have higher implied volatilities that OTM calls - it it more natural to speak of a "skew," which is indeed the convention we follow below.

${ }^{5}$ In this case it is more convenient to model volatility $v_{t}$ as the variance rather than standard deviation of returns. However, this convention by no means implies loss of generality and Ito's lemma can be easily used to transform the variance SDE $d V(t)=\ldots d t+\xi V(t)^{\alpha} d B(t)$ to a standard deviation equation $d \sigma(t)=$ $\ldots d t+\frac{1}{2} \xi \sigma(t)^{(2 \alpha-1)} d B(t)$. See also e.g. Alexander (2009).
} 
with $d Z_{1} d \bar{Z}_{1}=0$. Hence, plugging $\sqrt{v_{t}} d Z_{1}=d x_{t}+\frac{1}{2} v_{t}$ and $\phi=\sqrt{1-\rho^{2}}$ in the variance equation, we obtain

$$
d v_{t}=\alpha\left(v_{t}\right) d t+\rho \eta \beta\left(v_{t}\right)\left[d x_{t}+\frac{1}{2} v_{t}\right]+\phi \eta \beta\left(v_{t}\right) \sqrt{v_{t}} d \bar{Z}_{1}
$$

Equation (8) describes the evolution of instantaneous variance, whereby the only element of randomness, $d \bar{Z}_{1}$, is uncorrelated with the stochastic element in the dynamics of the underlying. We now prove the following fact which shows us how to relate the $v_{t}$ to Black-Scholes implied volatility.

Fact. Total implied Black-Scholes variance in the model described by equations (4) can be approximated by: $\sigma_{B S}^{2}(K, T) \approx \frac{1}{2} \rho \eta \beta\left(v_{0}\right) x_{T}$.

Proof. We provide a sketch of the proof which was originally suggested by Gatheral (2006). Note, first, that thanks to the orthogonalization,

$$
\mathbb{E}(v+d v \mid d x)=v+\alpha(v) d t+\rho \eta \beta(v)\left[d x+\frac{v}{2} d t\right]
$$

As shown independently by Derman and Kani (1998) and Dupire (2004), local variance can be expressed as conditional expectation of instantaneous variance $\sigma^{2}(K, T)=\mathbb{E}\left(v_{T} \mid S_{T}=\right.$ $K)$. Hence, for all $t$ close to the expiry $T$ we have the following approximation:

$$
\begin{aligned}
v_{l o c}(x, t) & =\mathbb{E}\left(v_{t} \mid x_{T}\right) \\
& \approx v_{0}+\left[\alpha\left(v_{0}\right)+\rho \eta \beta\left(v_{0}\right) \frac{v_{0}}{2}\right] t+\rho \eta \beta\left(v_{0}\right) x
\end{aligned}
$$

Knowing (approximately) the form of local variance we can obtain the Black-Scholes implied volatility (variance) as by integrating local variance along the most likely path $\tilde{x}_{t}$ of the underlying from $t$ to expiry date $T$. Since $\tilde{x}_{t}$ is approximately a straight line (in log space) given by $\tilde{x}_{t} \approx \frac{t}{T} x_{T}$, we can write

$$
\begin{aligned}
\sigma_{B S}^{2}(K, T) & \approx \frac{1}{T} \int_{0}^{T} v_{l o c}\left(\tilde{x}_{t}, t\right) d t \\
& \approx \ldots+\frac{1}{T} \int_{0}^{T} \rho \eta \beta\left(v_{0}\right) \tilde{x}_{t} d t \\
& \approx \ldots+\frac{1}{T} \int_{0}^{T} \rho \eta \beta\left(v_{0}\right) \frac{t}{T} x_{T} d t \\
& =\ldots+\frac{1}{2} \rho \eta \beta\left(v_{0}\right) x_{T},
\end{aligned}
$$

which is the desired approximation. 
Since $\sigma_{B S}^{2}(K, T) \approx \ldots+\frac{1}{2} \rho \eta \beta\left(v_{0}\right) x_{T}$, its derivative with respect to $x$ is

$$
\frac{\partial}{\partial x} \sigma_{B S}^{2}(x, t)=\frac{\rho \eta}{2} \beta\left(v_{0}\right) .
$$

Now, since we can interpret $\frac{\partial}{\partial x} \sigma_{B S}^{2}(x, t)$ as the slope of implied Black-Scholes volatility as a function of moneyness, we get an important corollary.

Corollary 1. Stochastic volatility models produce an implied volatility smile that is proportional to parametr $\eta$, or "volatility of volatility."

Translating this result into the language of our analysis, provides us with an important and testable theoretical insight, namely that SPX implied volatility skew should be correlated with the volatility of SPX volatility. And since the "volatility of volatility" should in turn be proportional to VIX, we ultimately arrive at the conclusion that SPX implied volatility skew itself should be proportional to VIX. Symbolically:

$$
\text { SPX skew } \propto \eta \propto \mathrm{VIX} \text {. }
$$

To verify this hypothesis we estimate a simple linear regression of VIX implied volatility on SPX skew. ${ }^{6}$ VIX implied volatility is defined as interpolated 1M ATM implied volatility back out of VIX options. In turn, SPX skew is parameterized as the difference in $1 \mathrm{M}$ SPX implied volatilities (interpolated) corresponding to strike levels equal to $90 \%$ and $120 \%$ ATMF, which span enough of the skew while still remaining liquid. We use daily data from Bloomberg for the period January 2010 through December 2013. The results of the estimation are shown in Figure 4. The fit between VIX 1M implied volatility (IV) and 1M SPX skew seems quite good $\left(R^{2}=0.66\right)$, particularly if contrasted with bid-ask spreads quoted on the market. For example, on December 30th 2013 the fitted VIX ATM IV stood at 0.59 vs. 0.54 quoted on the market with a bid-ask spread of about 6 vol points.

\subsection{Term structure of VIX implied volatility}

The preceding section documents a clear positive relationship between the skew in SPX implied volatilities and the 1M ATM VIX implied volatility. So far, this allows us to "price" VIX options but only those with exactly $1 \mathrm{M}$ to expiry and strike equal to the forward price. Ideally, we would like to be able to price any VIX options and for that we need to parameterize the whole volatility surface - i.e. relate SPX skew to VIX implied volatilities for different strikes and expiries. In this section we go one step in that direction by suggesting a natural

\footnotetext{
${ }^{6}$ To the extent that skewness is thought as "slope" of the SPX smile, it could also be combined with some measure of "convexity" to capture the properties of the SPX volatilities more fully. We owe this point to Arun Verma.
} 
Figure 4: Correlation of VIX implied volatility and SPX volatility skew (January 2010December 2013)

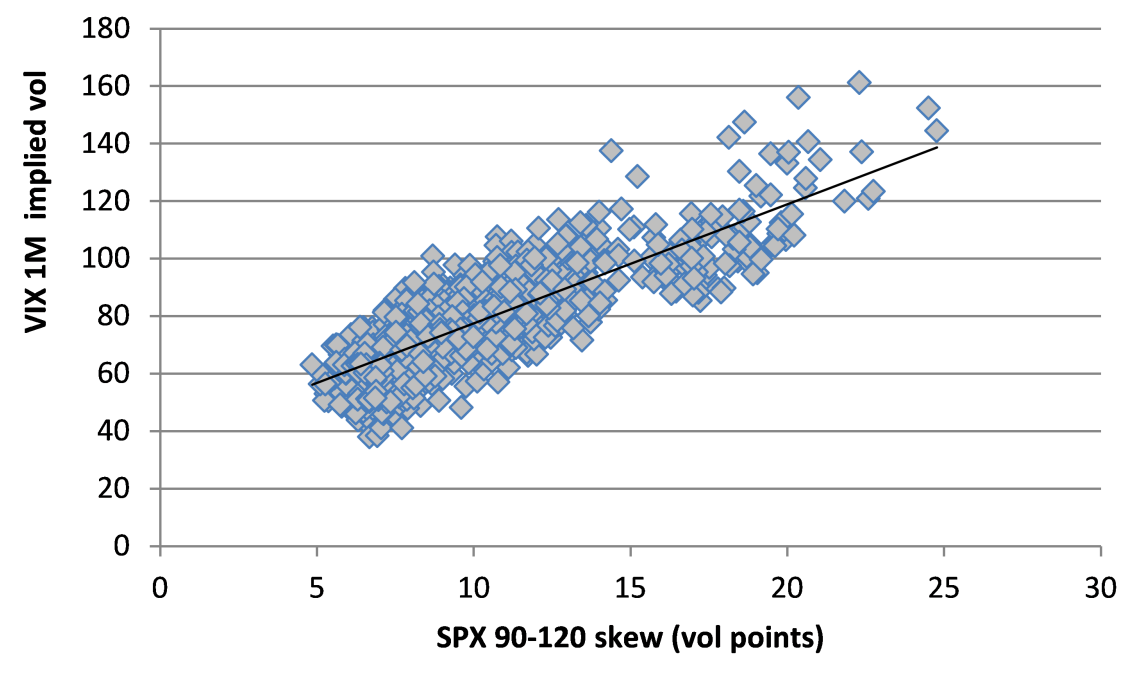

Source: Bloomberg data. VIX implied volatility is the interpolated 1M volatility implied out of VIX options. SPX skew is defined as the difference between 1M SPX implied volatilities corresponding to $120 \%$ and $90 \%$ ATMF. OLS regression has the form VIX $1 \mathrm{M}$ IV $=4.1434 \times \mathrm{SPX}$ skew +36.039 with $R^{2}=0.6649$.

and simple model for the term structure of VIX ATM implied volatilities, while the next section deals with the parameterization of the whole VIX implied volatility smile.

Once again we start with our general stochastic volatility model (4). In order to be able to say something specific about the term structure of implied volatility we need to impose some assumptions on the functional forms of $\alpha(\cdot)$ and $\beta(\cdot)$. Since our goal here is not to build a formal pricing model per se, but rather to derive some theoretically grounded intuitions, we consider a popular parameterization due to Cox, Ingersoll Jr, and Ross 1985 with $\alpha(v)=\lambda(\bar{v}-v)$ and $\beta(v)=1$. Parametr $\bar{v}>0$ stands for the long run average volatility and $\lambda>0$ determines the spead of mean-reversion. ${ }^{7}$ Equation (8) thus becomes:

$$
d v_{t}=\lambda(\bar{v}-v) d t+\rho \eta\left[d x_{t}+\frac{1}{2} v_{t}\right]+\phi \eta \sqrt{v_{t}} d \bar{Z}_{1} .
$$

Unconditional expectation of instantaneous variance at time $t$ has the form $v_{t}=\left(v_{0}-\right.$ $\bar{v}) \exp (-\lambda t)+\bar{v}$. Hence, total variance $w_{t}$ realized up to $t$, as an integral of instantaneous variances, is given by:

$$
w_{t}=\int_{0}^{t} v_{s} d s=\left(v_{0}-\bar{v}\right) \frac{1-\exp (-\lambda t)}{\lambda}+\lambda t .
$$

\footnotetext{
${ }^{7}$ Cox, Ingersoll and Ross use such specification to model the term structure of interest rates, but in Heston (1993) it is used for modeling the dynamics of volatility.
} 
Figure 5: Comparison of the empirical VIX ATM 3M implied volatility with model fit.

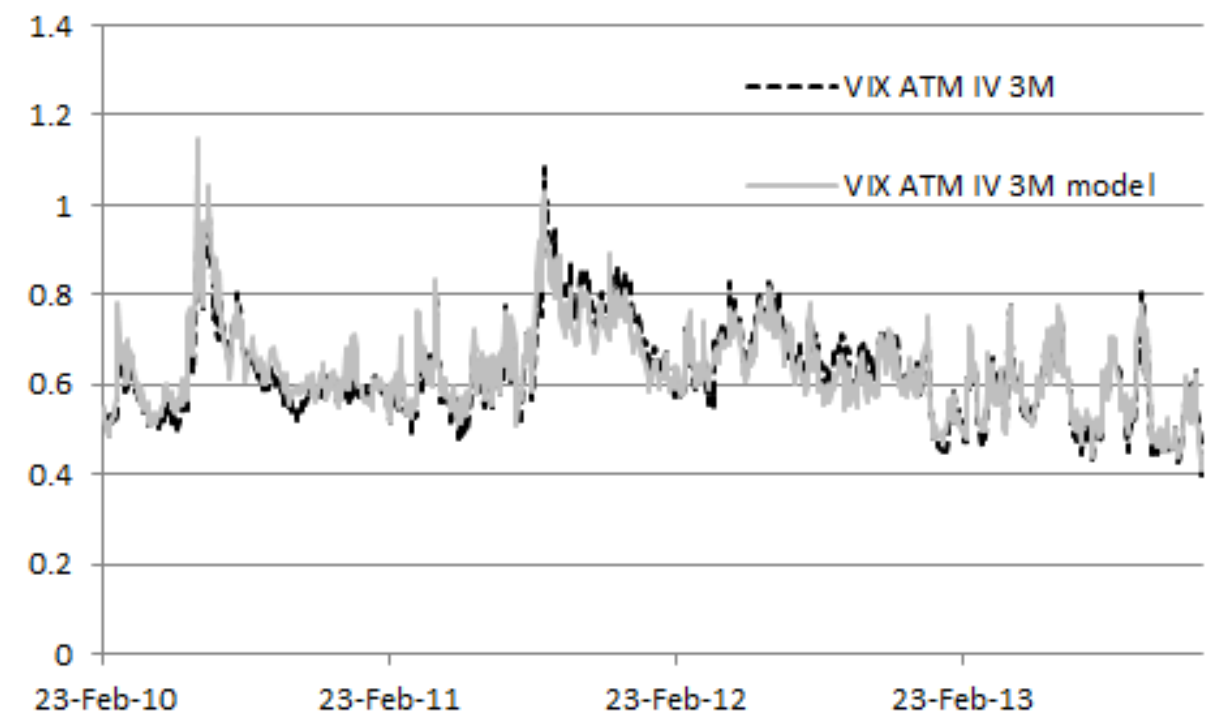

Source: Bloomberg data. The model used in the calculations has the form $\sigma(t)=0.45+\left(\sigma_{1 M}-0.45\right) \exp (3.8 \times$ $(1 M-t))$.

Moreover, Gatheral (2006) shows that Black-Scholes implied volatility, determined as before by integrating local variance along the most likely path of the underlying, will have an analogous, exponentially decaying form. Both of these theoretical arguments suggest that it is natural to express VIX ATM implied volatility as a function of time in the following way:

$$
\sigma(t)=\Sigma+\left(\sigma_{1 M}(t)-\Sigma\right) e^{(1 M-t) \lambda}
$$

with $\Sigma$ being long-run average volatility, and $\lambda$ the speed with which $\sigma(t)$ approaches $\Sigma$. The monthly VIX volatility $\sigma_{1 M}$ that we have chosen as a benchmark (determined on the basis of SPX 1M skew, see Figure 4) plays the role of $v_{0}$ parameter in equation (14). Parameters $\Sigma$ and $\lambda$ are estimated using least squares by fitting equation (15) to implied volatility time series VIX ATM 1M, 2M and 3M from January 2010 through December 2013 r. (daily data). The estimated values are $\Sigma=0.45$ and $\lambda=3.8$. Mean absolute error was 2.3 vol points for the $2 \mathrm{M}$ term and 2.7 points for the $3 \mathrm{M}$ term, i.e. well within the average bid-ask spread. Figure 5 shows the fitted $3 \mathrm{M}$ volatilities against their empirical levels.

\subsection{VIX volatility smile}

The results thus far allow us to relate the volatility skew observed in SPX options to VIX ATM volatility of any term. The last step on the way to full parameterization of VIX volatility surface consists in finding an appropriate model for VIX implied volatility as a 
function of strike, or moneyness. A popular smile model is the "stochastic alpha beta rho," or SABR, suggested by Hagan, Kumar, Lesniewski, and Woodward (2002). The model has the following dynamics ${ }^{8}$ :

$$
\begin{aligned}
d f_{t}^{T} & =\left(f_{t}^{T}\right)^{\beta_{T}} \sigma_{t}^{T} d Z_{t}^{T} \\
d \sigma_{t}^{T} & =\eta^{T} \sigma_{t}^{T} d W_{t}^{T} \\
d Z_{t}^{T} d W_{t}^{T} & =\rho d t
\end{aligned}
$$

where $f_{t}^{T}$ is a futures contract with expiration date $T, \eta$ is, as before, the volatility of volatility, while $\rho$ denotes the constant correlation of processes $d Z$ and $d W$. Parameters $\beta$ and $\rho$ control the slope of the volatility curve. In the particular case when $\beta=0$, equation (16) reduces to a Bachelier model, albeit with stochastic volatility, while for $\beta=1$ we get lognormal dynamics of the underlying well known from the Black and Scholes model. By design, SABR describes the implied volatility curve as a function of strike for a given exercise date $T$. In other words, parameters $\beta, \rho, \eta$ are constants, specific to a particular expiry of the futures contract - which justifies our use of superscript $T$ in equations (16). In that sense, SABR is not a model of the whole volatility curve, but rather a tool to handle smiles for particular expiries $T$ - for each $T$ a separate volatility curve $\sigma^{T}(K)$ needs to be fitted, characterized by parameters $\left(\beta^{T}, \rho^{T}, \eta^{T}\right)$. As explained by Rebonato, McKay, and White (2011), this restrictiveness should not be held against SABR, which should simply be treated as a tool for estimating the values $\left(\beta^{T}, \rho^{T}, \eta^{T}\right)$. The latter, in turn, can be thought of as parameters of some more general model of the whole volatility surface.

One virtue of the SABR model - which comes in particularly handy in this context - is that it yields exact closed-form algebraic formulas for the implied volatility as a function of strike, i.e. a closed-form formula for the implied volatility smile. Specifically, Hagan, Kumar, Lesniewski, and Woodward (2002) use singular perturbation techniques on (16) to obtain the price for a European call option $C_{t}$ which turns out to be given by Black's formula:

$$
C_{t}=\exp (-r T)\left[f_{t} N\left(d_{+}\right)-K N\left(d_{-}\right)\right]
$$

with

$$
d_{ \pm}=\frac{\log (f / K) \pm \frac{1}{2} \sigma_{B}^{2} T}{\sqrt{\sigma_{B}^{2} T}}
$$

and implied volatility $\sigma_{B}(f, K)$ given by

\footnotetext{
${ }^{8}$ In Hagan, Kumar, Lesniewski, and Woodward (2002) the stochastic volatility parameter is denoted by $\alpha$. However, to avoid confusion, we follow below the well-established convention of denoting volatility by the Greek letter $\sigma$ rather than $\alpha$.
} 


$$
\begin{aligned}
\sigma_{B}(f, K)= & \frac{\sigma}{(f K)^{(1-\beta) / 2}\left\{1+\frac{(1-\beta)^{2}}{24} \log ^{2}(f / K)+\frac{(1-\beta)^{4}}{1920} \log ^{4}(f / K)+\ldots\right\}} \cdot \frac{z}{\chi(z)} \cdot \\
& \cdot\left\{1+\left[\frac{(1-\beta)^{2}}{24} \frac{\sigma^{2}}{(f K)^{1-\beta}}+\frac{1}{4} \frac{\rho \beta \eta \sigma}{(f K)^{(1-\beta) / 2}}+\frac{2-3 \rho^{2}}{24} \eta^{2}\right] T+\ldots\right\}
\end{aligned}
$$

Here $z=\frac{\eta}{\sigma}(f K)^{(1-\beta) / 2} \log (f / K)$ and $\chi(z)=\log \left(\sqrt{1-2 \rho z+2 z^{2}}+z-\rho\right)-\log (1-\rho)$. In the specific case of ATM implied volatility the formula reduces to

$$
\sigma_{A T M}=\frac{\sigma}{f^{1-\beta}}\left\{1+\left[\frac{(1-\beta)^{2}}{24} \frac{\sigma^{2}}{f^{2-2 \beta}}+\frac{1}{4} \frac{\rho \beta \eta \sigma}{f^{1-\beta}}+\frac{2-3 \rho^{2}}{24} \eta^{2}\right] T+\ldots\right\}
$$

Generally speaking, the calibration of the SABR model consists in finding such a combination of parameters $\sigma, \beta, \rho, \eta$ that for given expiry and moneyness, $T$ and $f / K$, implied volatilities calculated according to formula (19) are as close as possible to values quoted on the market. Since, as pointed out by Hagan, Kumar, Lesniewski, and Woodward (2002), market smiles can be fit equally well with any specific value of the exponent $\beta$. This implies that $\beta$ - which along with $\rho$ is responsible for a downward sloping skew in the smile - should not be determined in the calibration process, as that would amount to "fitting the noise." However, $\beta$ can be estimated instead by OLS regression using the fact that

$$
\log \left(\sigma_{A T M}\right) \approx \log (\sigma)-(1-\beta) \log (f)
$$

which is obtained by taking the natural logarithm of both sides in equation (20). In practice, $\beta$ might just as well be determined on the basis of general "aesthetic" considerations, depending on the market in question (FX, interest rates etc.). Given that it seems natural to assume that volatility should have a lognormal distribution, and taking into account numerical stability concerns we opt for $\beta=0.999$. The subsequent steps of the calibration are as follows:

- for a given $T$ find the corresponding implied at-the-money volatility, $\sigma_{A T M}{ }^{9}$;

- based on $\sigma_{A T M}$ and $T$ solve (20) to find $\sigma^{T}$;

- determine the remaining two parameters, $\rho$ and $\eta$, numerically by minimizing $\left(\rho^{T}, \eta^{T}\right)=$ $\arg \min _{\beta, \rho} \sum_{i}\left[\sigma_{i}^{M}-\sigma_{B}\left(f, K_{i}, \sigma^{T}, \rho, \eta\right)\right]^{2}$, with $\sigma_{i}^{M}$ being the implied volatilities observed in the market.

\footnotetext{
${ }^{9}$ If $\sigma_{A T M}$ is not available for a given term, we use cubic splines interpolation. See e.g. Hagan and West (2008).
} 
Figure 6: Comparison of empirical VIX implied volatilities with SABR fit (January 2014 options as of December 30, 2013).

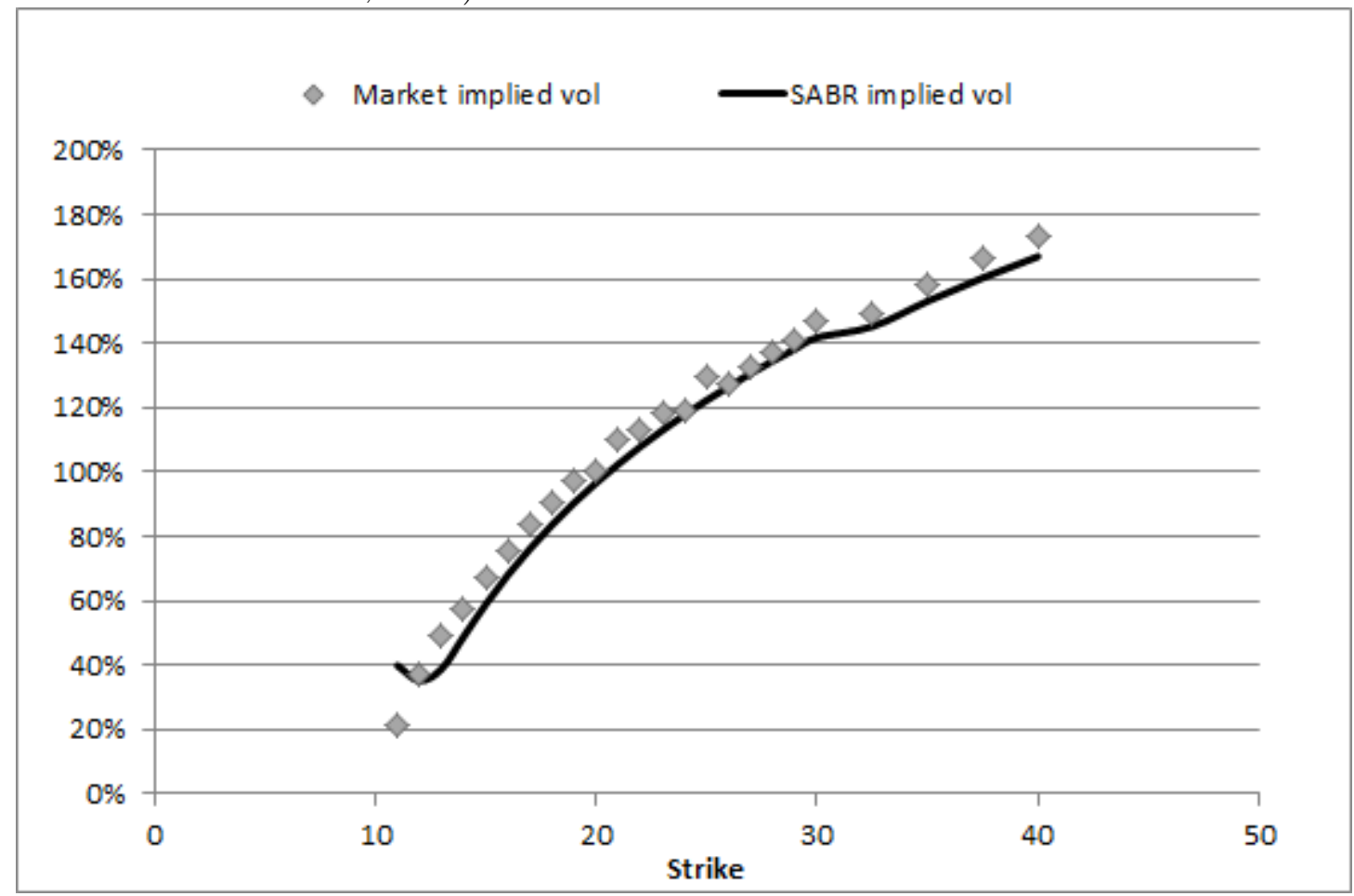

SABR implied volatility parameterized by $\sigma=0.411, \beta=0.999, \rho=0.666, \eta=3.644$.

We repeat this procedure for all option expiries available in the market. As of December 31, 2013 VIX options were available with six expiries: in January (22 days), February (50 days), March (77 days), April (106 days), May (141 days) and June (169 days). Figure 6 shows one such calibration for options with expiration date January 22, 2014. . Evidently, model fit is quite satisfactory. Interestingly, the correlation parameter $\rho$ does not seem to vary much with expiration time, while $\nu$ appears to decay with $T$ roughly as $\sim 0.5 T^{-0.75}$. Both observations are close to those derived by Gatheral (2008).

By combinig all the results described above we can finally fully parameterize the VIX volatility surface:

Corollary 2. For any $T$, the VIX volatility surface can be characterized by the following set of parameters: 
Figure 7: Comparison of empirical VIX implied volatilities with heuristic model fit (February 2014 options as of December 30, 2013).

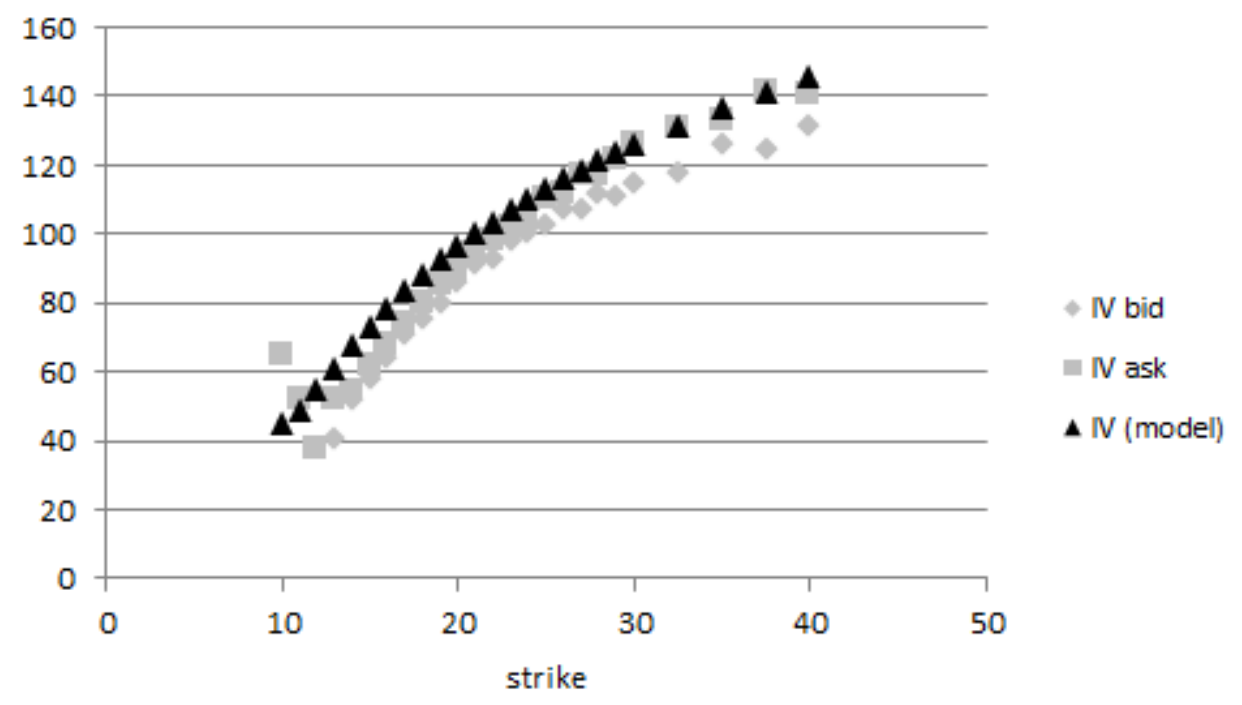

Source: Bloomberg data. IV bid, IV ask and IV (model) denote bid, ask and theoretical model-based implied volatility.

$$
\begin{aligned}
\sigma_{A T M}^{1 M} & =4.6 \times S P X 1 M \text { skew }_{90-120}+29.7 \\
\sigma_{A T M}^{T} & =0.45+\left(\sigma_{A T M}^{1 M}-0.45\right) e^{3.8 \frac{(30-T)}{365}} \\
\beta^{T} & =0.999 \\
\rho^{T} & =0.71 \\
\eta^{T} & =0.5 \times T^{-0.75}
\end{aligned}
$$

Using formulas from Corollary 2 we can estimate implied volatilities for VIX options with any strike and maturity. In other words, Corollary 2 provides a set of simple rules for pricing VIX options. Figures 7 and 8 show the theoretical values of VIX implied volatilities for two randomly chosen dates (June 6, 2013 and December 30, 2013) and two expiries (104 days and 50 days). In both cases, the theoretical prices calculated with our heuristic rules are reasonably close to market levels. The somewhat better fit obtained for the 50-day options can be attributed to the fact that SABR works best for relatively short expirations (as there is no mean reversion in the model). 
Figure 8: Comparison of empirical VIX implied volatilities with heuristic model fit (September 2013 options as of June 6, 2013).

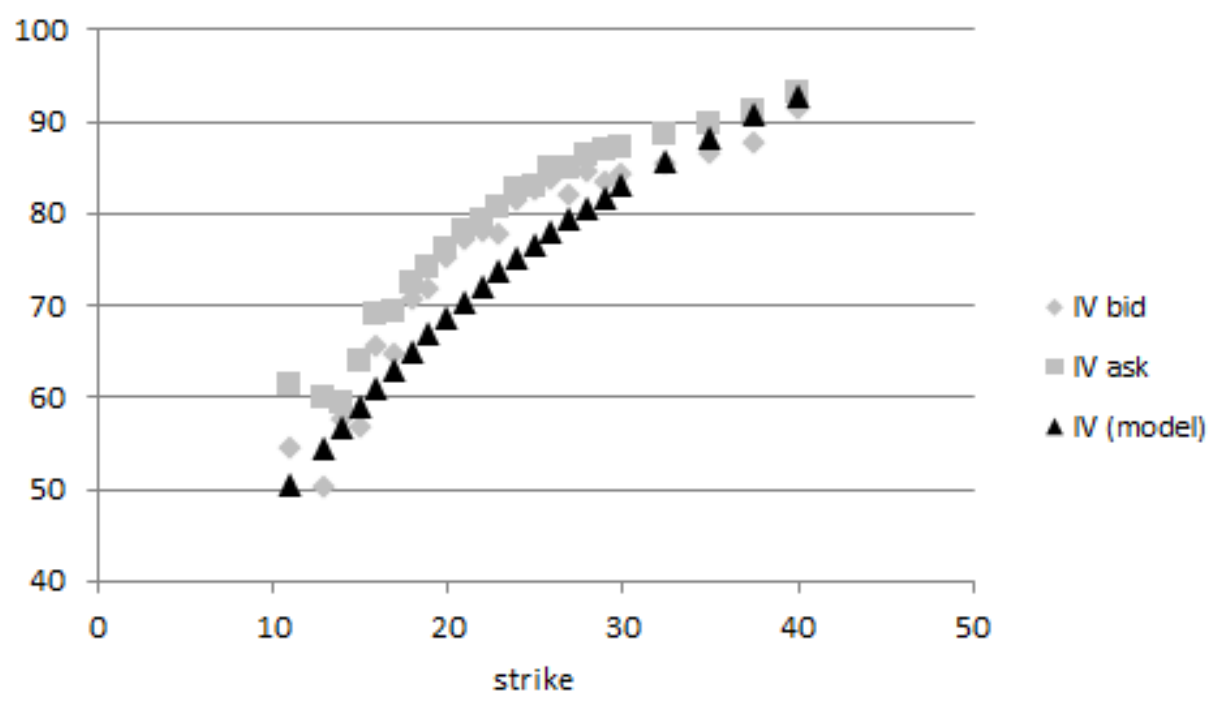

Source: Bloomberg data. IV bid, IV ask and IV (model) denote bid, ask and theoretical model-based implied volatility.

\section{Conclusions}

The goal of this article was to find a set of theoretically-grounded, but easy to implement heuristic rules allowing for the parameterization of the full VIX volatility surface. The rules we describe are based on the connection between the skew observed in S\&P 500 options volatilities and the volatility of the VIX, and despite apparent simplicity, yield prices that are relatively close to those observed in the market. Clearly, the solution we propose cannot be a substitute for a fully-fledged formal pricing model for VIX options. Rather, it should be treated as a sort of "calculator," which uses the prices of more liquid instruments (options on S\&P 500) to estimate the prices of less liquid instruments. A natural extension of the approach we suggest above could consist in finding similar heuristics for even less liquid options on other volatility indices. Our results could also be developed further on the theoretical side e.g. by trying to find a more formal description of the term structure of the volatility of volatility parameter $\eta$ in stochastic volatility models. As we have argued above, our choice for the functional form $\eta(T)=0.5 T^{-0.75}$ finds some backing in the literature, but it should be borne in mind that - at least in our case - it was based on very few observations. A more thorough theoretical investigation of the term structure of $\eta$ would provide additional backing for the universal applicability of our results. 


\section{References}

Alexander, C. (2009): Market Risk Analysis, Pricing, Hedging and Trading Financial Instruments, vol. 3. Wiley. com.

BlaCK, F. (1976): "The pricing of commodity contracts," Journal of financial economics, $3(1), 167-179$.

Black, F., And M. S. Scholes (1973): "The Pricing of Options and Corporate Liabilities," Journal of Political Economy, 81(3), 637-54.

Carr, P., And D. Madan (2001): "Towards a Theory of Volatility Trading," in Volatility, ed. by R. Jarrow, pp. 417-427. Risk Publications.

Carr, P., And L. Wu (2006): "A Tale of Two Indices," The Journal of Derivatives, 13(3), $13-29$.

CBOE (2003): "The CBOE Volatility Index - VIX," http://www.cboe.com/micro/vix/vixwhite.pdf.

Cont, R., And T. Kokholm (2011): “A consistent pricing model for index options and volatility derivatives," Mathematical Finance.

Cox, J. C., J. E. Ingersoll JR, and S. A. Ross (1985): "A theory of the term structure of interest rates," Econometrica: Journal of the Econometric Society, pp. 385-407.

Derman, E., K. Demeterfi, M. Kamal, and J. Zou (1999): "More Than You Ever Wanted To Know About Volatility Swaps," Quantitative strategies research notes, Goldman Sachs.

Derman, E., And I. KAni (1998): "Stochastic implied trees: Arbitrage pricing with stochastic term and strike structure of volatility," International Journal of Theoretical and Applied Finance, 1(01), 61-110.

Deshpande, M., R. Bhatia, and A. Goyal (2011): "Hedging \& Alpha Strategies with VIX Derivatives," Global Derivatives USA 2011.

Dupire, B. (2004): "A unified theory of volatility," Working paper, Paribas Capital Markets.

Gatheral, J. (2006): The Volatility Surface - A Practitioner's Guide. John Wiley \& Sons Ltd.

(2008): "Consistent modeling of SPX and VIX options," . 
Grünbichler, A., And F. A. LongstafF (1996): "Valuing futures and options on volatility," Journal of Banking \& Finance, 20(6), 985-1001.

Hagan, P. S., D. Kumar, A. S. Lesniewski, and D. E. Woodward (2002): "Managing smile risk," Wilmott Magazine, pp. 84-108.

Hagan, P. S., And G. West (2008): "Methods for constructing a yield curve," WILMOTT Magazine, May, pp. 70-81.

Heston, S. L. (1993): "A closed-form solution for options with stochastic volatility with applications to bond and currency options," Review of financial studies, 6(2), 327-343.

Hull, J., AND A. White (1987): "The pricing of options on assets with stochastic volatilities," The journal of finance, 42(2), 281-300.

Lin, Y.-N., And C.-H. Chang (2010): "Consistent modeling of S\&P 500 and VIX derivatives," Journal of Economic Dynamics and Control, 34(11), 2302-2319.

Merton, R. C. (1973): "Theory of Rational Option Pricing," Bell Journal of Economics, $4(1), 141-183$.

Moran, M. T., And S. DAsh (2007): "VIX futures and options: Pricing and using volatility products to manage downside risk and improve efficiency in equity portfolios," The Journal of Trading, 2(3), 96-105.

Rebonato, R., K. McKay, and R. White (2011): "The SABR/LIBOR Market Model: Pricing, calibration and hedging for complex interest-rate derivatives," .

SEPP, A. (2008): "VIX option pricing in a jump-diffusion model," Risk Magazine, pp. 84-89.

Whaley, R. E. (1993): "Derivatives on Market Volatility: Hedging Tools Long Overdue," Journal of Derivatives, 1, 71-84.

Wilmott, P. (2006): Paul Wilmott On Quantitative Finance. John Wiley \& Sons Ltd. 


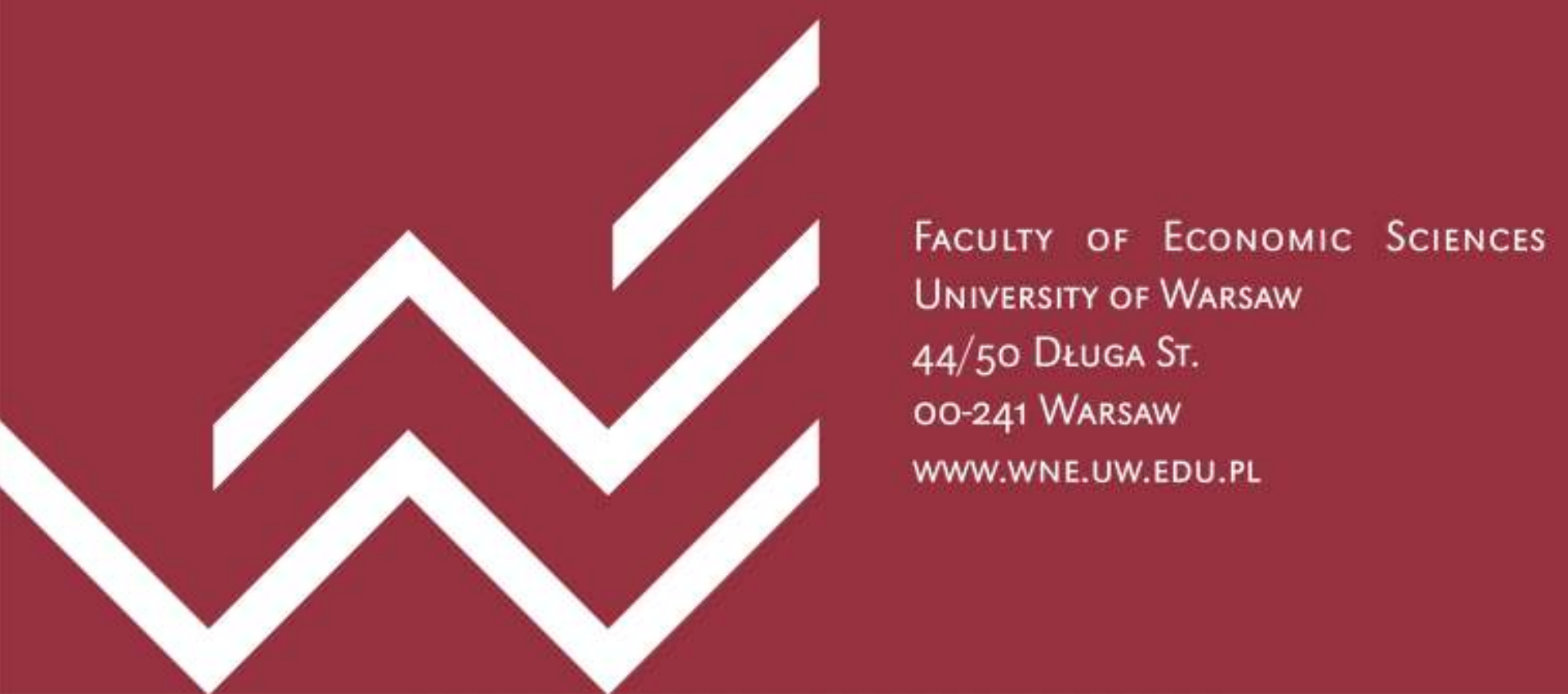

